time. As he did not improve, for the sake of a holiday, he paid a visit to his daughter in Essex. Shortly after his arrival he developed difficulty in swallowing and soon had to limit his food to semi-solids and finally liquids. The latter, however, frequently regurgitated through the nose.

After he had been in Essex about three days he was seen by one of us and again carefully examined. He was then well nourished and when sitting down looked in good health except for his ptosis. He complained of weakness; but on examination it was found that he could fairly well perform any movement of the limbs or trunk. Any such movement, however, was almost immediately exhausted; for instance, he could rise from his chair fairly briskly for a man of his age; a second attempt to do this involved great effort and a third attempt was quite unsuccessful. There was no muscular wasting and the electrical irritability of the muscles was not examined. There was no loss of sensation in any part of the body, nor was there any incoördination, tremor, or involuntary movement of any kind. The abdominal and plantar reflexes were normal. The kneejerk was at first somewhat exaggerated but became partially exhausted on repeated stimulation. As far as could be determined, however, the knee-jerk could not be absolutely abolished. There was no clonus. There was no ocular palsy, but some nystagmoid jerking occurred on lateral deviation of the eyes, which jerking increased if the lateral deviation was prolonged. There was no history of diplopia. Bilateral ptosis was present to a degree sufficient about half to cover the pupils, but the ptosis increased if the patient attempted to read. The pupils were equal and the pupillary and conjunctival reflexes were normal. There appeared to be no weakness of the muscles of mastication or of the muscles of the face. The patient's voice was fairly strong after a rest of half an hour or so, but aphonia developed after a few minutes' conversation. He was unable to swallow solid food. The pharyngeal reflex could not be obtained. Liquids regurgitated through the nose towards the end of a meal. The heart and lungs were normal except that the aortic second sound was louder than natural. His arteries were normal for his age. The patient had full control of his rectum and bladder. At this time the urine contained both sugar and albumin in moderate amounts. The patient rapidly grew worse and returned to Plymouth on June 27th. On the 29th difficulty of swallowing became more pronounced and it increased daily until his death on July 13th.

No necropsy was obtained in this case, but there appears to be little doubt that our patient suffered from myasthenia gravis or asthenic bulbar paralysis. The unusual features to which we would draw attention are the patient's age, the exceptionally acute course of the disease, and the association with diabetes. From previously reported cases (about 70 in number) it would appear that the disease belongs to the third decade of life, and so far as we have seen in the literature no case is on record of such a patient who has reached the seventh decade. Erb's first case-the oldest at present on record-was 55 years of age.

The present case ran an exceptionally acute course. The only undoubted case that has run a more acute course is that recorded by Widal and Marinesco; their patient lived only 14 days after the onset. The association with diabetes in our case appears to have been somewhat intimate since the myasthenia set in almost as soon as the patient relaxed his diabetic diet. So far as this observation goes it is in favour of the prevalent view that myasthenia gravis is due to the effect of some toxic agent upon the peripheral neurons.

\section{MILK OR WHEY IN ENTERIC FEVER?}

BX ARTHUR TREGHLLES PRIDHAM, M.B. LOND., HOUSE PHYSICLAN AT ST. BARTHOLOMEW'S HOSPITAL.

In The Lancet of Nov. 2nd, 1901, p. 1182, Mr. Prideaux G. Selby of Teynham, Kent, published a paper concerning the treatment of enteric fever by whey instead of milk as a taple food, his death-rate for 73 cases being 2.7 per cent. Since that time Dr. S. J. Gee of St. Bartholomew's Hospital has treated four cases entirely, and one case partially, by this method. All did well, but one case perhaps deserves notice on account of its severity and the favourable issue under what is more or less a new departure in treatment, though I believe that Dr. Norman Moore has for some time past treated his cases of enteric fever chiefly on whey. The patient in question was a man, aged 23 years, a fish-porter, and previously to this illness he had been in good health. On Nov. 3rd, 1901, he caught cold and suffered from headache, and on the 10th he took to his bed. On the 16th he was admitted into St. Bartholomew's Hospital, with the history that for the previous week he had been feverish and his bowels constipated. On admission his tem. perature was $100 \cdot 5^{\circ} \mathrm{F}$. ; his pulse was 84 per minute, soft and regular; his radial artery was thickened; his urine was of specific gravity 1025, had an acid reaction, and presented no abnormality; and his tongue was dry and brown. Examination of his heart and lungs showed nothing abnormal. His abdomen was not distended or tender; his spleen could just be felt. On the 17th his blood gave a well-marked Widal's reaction. On Dec. 2nd there were many typical enteric fever spots on his abdomen and chest. His evening temperature was usually from $103^{\circ}$ to $104^{\circ}$, and his pulse was from 110 to 120 per minute and very soft; he was sponged night and morning. He was taking four ounces of brandy and a small quantity (about half a pint) of beef essence; otherwise he had nothing but whey, of which he consumed from three to four pints in the 24 hours. On Dec. 4th there were some indications of heart failure and the first sound became faint. The patient therefore began to take four-minim doses of liquor strychniæ, given at tirst three times a day and afterwards every six hours. On the 6 th his pulse was very soft and dicrotic and he had occasional shivering fits. His bowels were open about three times daily; on the 6 th the motions were stained with blood, and he passed one very large black slough measuring one inch by half an inch, together with several small sloughs; they were of considerable thickness, showing the ulceration to be very deep. His abdomen presented no distension and was natural in appearance. On the 9th several large blood-stained sloughs were passed. On the 10th the man was very ill and the brandy was gradually increased till he now took eight ounces. About $3 \mathrm{~A}$. M. on the 12 th, which was approximately the fortieth day of the disease, there was copious hæmorrhage from the bowel amounting to at least one and a half pints; the man was very ill, but he quieted down with morphia given hypodermically. On the 13th the patient was so collapsed and blanched that Dr. Gee gave an unfavourable prognosis. There was, however, no delirium or distension of the abdomen. On the 16th the patient was very restless, he passed urine frequently, and was with great difficulty made to sleep by hypodermic injec. tions of morphia, hot sponging, and paraldehyde. On the 17th his urine was alkaline and contained much pus and the bacillus typhosus; he was given seven grains of urotropine three times a day. On the 18th he was better and the urine was clearing. On the 19th there was an obvious improvement. His bowels were opened twice, the motions containing no blood or sloughs. His tongue was cleaner and for the first time since the hæmorrhage he had strength to put it out when asked to do so. On the 20th the man was better. His temperature was intermitting well and his urine contained no pus. On the 21st (the forty-ninth day of the disease) his temperature was normal, his subsequent convalescence was uninterrupted, and the brandy was gradually reduced. He continued to take rothing but whey until the 31st, when he began to take milk. He was very emaciated, but not more so than might have been expected from the severity of the disease.

The most noticeable points in the case were the following. 1. Distension of the abdomen was entirely absent. Another patient with enteric fever who was taking half a pint of milk with his whey showed more distension on the third day after admission than this patient did at any time throughout his illness. From the size and thickness of the sloughs I am convinced that had the patient's intestines been distended with gas when he passed these sloughs perforation must have followed. 2. There was an absence of delirium almost throughout the illness, except for a short time on one or two occasions. 3. There was an absence of serious diarrhœa in spite of the severe ulceration; the bowels were loose but never became troublesome. It is probable, as suggested by Mr. Selby, that these three symptoms are often much aggravated by, or due to, toxins formed by decomposing milk curd. 4. With regard to the state of the mouth and tongue it did not correspond to Mr. Selby's experience and differed little from than of an enteric fever patient treated with milk, being dry and cracked and difficult to keep clean.

St. Bartholomew's Hospital, E.C. 